\title{
THE EFFECT OF BENEMID (P-[DI-N-PROPYLSULFAMYL]- BENZOIC ACID) ON URIC ACID METABOLISM IN ONE NORMAL AND ONE GOUTY SUBJECT ${ }^{1,2}$
}

\author{
BY CHARLES BISHOP, ROYDEN RAND, AND JOHN H. TALBOTT
}

\author{
(From the Departments of Medicine and Biochemistry of the University of Buffalo Medical \\ School and the Buffalo General Hospital, Buffalo, N. Y.)
}

(Submitted for publication February 23, 1951; accepted May 28, 1951)

Benzoic acid derivatives such as carinamide (4carboxy-phenyl-methane-sulfonanilide) have been reported to be effective uricosuric agents, presumably by blocking renal tubular resorptions of uric acid. Benemid (p-[di-n-propylsulfamyl]benzoic acid), another compound of this type, has recently become available. In patients with tuberculosis undergoing treatment with PAS ( $\mathrm{p}$-aminosalicylic acid) the simultaneous administration of Benemid tended to prevent the rapid renal excretion of the PAS. Since this effect was presumably due to some blocking of renal tubular activity, it became of interest to test the effect of Benemid on uric acid excretion.

The present study was carried out on two subjects. One was an idiopathic hyperuricemic subject (J. H. T.) who appeared from previous studies to have a normal uric acid metabolism; the other subject (W.S.) was known to be suffering from gouty arthritis and had been studied previously in this laboratory.

Before and after a course of Benemid therapy, $\mathrm{N}^{15}$ labeled uric acid was injected intravenously for the determination of uric acid pool size and turnover rate. Uric acid excretion was followed quantitatively by the isotope dilution method.

\section{EXPERIMENTAL PROCEDURES}

Each subject was injected intravenously with $\mathrm{N}^{15}$ labeled uric acid (25-50 mg, 16 atom per cent excess) and urine samples were collected for several days. The subject then began the daily ingestion of $2 \mathrm{gm}$ of Benemid. On the third day of this regimen, labeled uric acid was

\footnotetext{
1 Part of the data from this paper may be submitted by Royden Rand to the Graduate School of the University of Buffalo in partial fulfillment of requirements for the degree of Doctor of Philosophy in the Department of Biochemistry.

2 This work has been carried out under grants from the Weed Foundation, Buffalo, New York and the National Institutes of Health, Public Health Service.
}

again injected. Benemid ingestion was continued until the end of the study.

The determination of uric acid pool size and turnover rate employed the same materials and techniques as previously reported (1). In addition, an aliquot of each urine sample or combination of samples was taken for quantitative uric acid determination by the isotope dilution method. To this aliquot were added $25-50 \mathrm{mg}$ of purified $\mathrm{N}^{15}$ uric acid of approximately 1 atom per cent excess dissolved in dilute sodium hydroxide solution. The molecules of added uric acid were presumed to mix completely with the molecules of uric acid present in the aliquot. A representative sample of this mixture of uric acid molecules was precipitated and its isotope concentration compared with that of a sample of uric acid precipitated from the same urine without augmentation. The amount of uric acid present in the original urine was calculated by the isotope dilution principle.

From standard solutions of uric acid it was demonstrated that this method under conditions comparable to those used for urine, gave results accurate to within 2 per cent. This implied that for a daily excretion of 800 $\mathrm{mg}$ the determination would be accurate to within \pm 16 mg. It was believed that such results were considerably better than could be expected from any of the usual methods for the quantitative determination of urinary uric acid. Furthermore, the material determined as uric acid was uric acid, not a mixture of uric acid and nonuric acid chromogen as in the usual colorimetric procedures.

Serum uric acid concentrations were determined by the method of Folin (2). Since these values were only used as a clinical guide and not as the basis of further calculation, it is not so necessary that they be highly specific for uric acid.

\section{RESULTS}

Figure 1 is a graph of the isotope concentration of the urinary uric acid of the non-gouty subject (J. H. T.) in the period before the ingestion of Benemid. On day 160, Benemid ingestion was begun and was continued through day 166. Figure 2 shows the isotope concentration after the injection of labeled uric acid on day 163 and during the period of Benemid ingestion. The uric acid pool size in the control period was calculated by assum- 


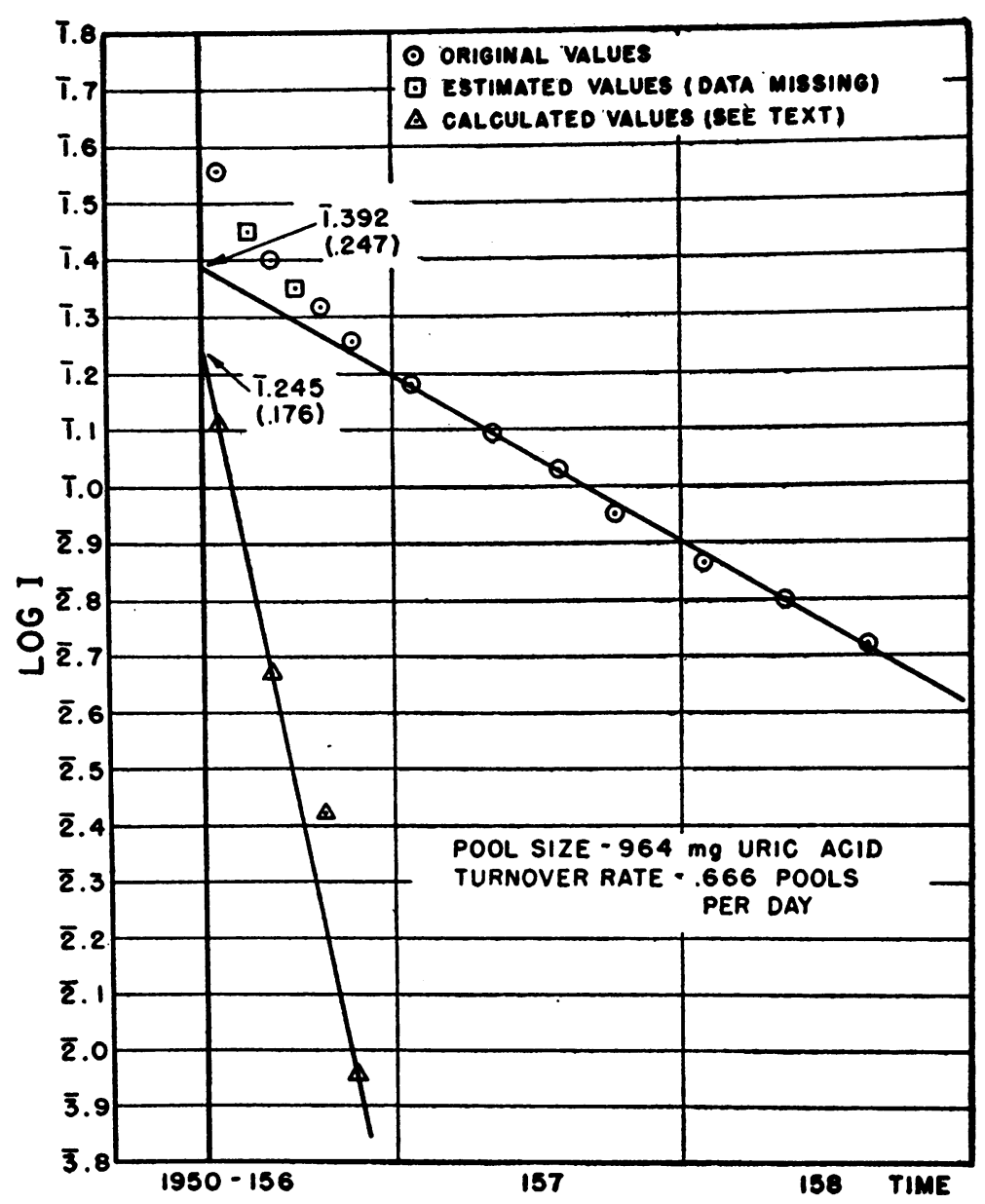

Fig. 1. Isotope Concentration of Urinary Uric Acid of Subject J. H. T. BEFORE BeNemid Ingestion

ing a second additive process according to the method previously outlined (1). This was unnecessary in the Benemid period. In the control period the amount of body uric acid with which the injected uric acid immediately equilibrated was calculated to be $964 \mathrm{mg}$. The turnover rate was .666 pools per day. After Benemid therapy the immediate pool size dropped to $466 \mathrm{mg}$, and the turnover rate rose to 2.457 pools per day. These results are consistent with the interpretation that Benemid is an effective tubular blocking agent and inhibits the resorption of uric acid. Under such circumstances some uric acid from the body pool should be excreted, leading to a decrease in the pool size and an increase in the turnover rate. (If the rate of synthesis of body uric is constant then a decrease in the pool size is equivalent to an increase in the turnover rate in pools per day.)
In Figure 3, the daily uric acid output of the same subject has been represented by a bar graph. On the same graph has been plotted the serum uric acid concentration. The fall in serum uric acid concentration and the rise in urinary uric acid excretion during Benemid therapy is apparent.

The mean daily uric acid excretion during an 11 day control period which included all days of urinary collection before and after Benemid administration was $830 \mathrm{mg}$. The "extra" uric acid excreted during the seven days on Benemid was $2705 \mathrm{mg}$. This was several times the magnitude of the $498 \mathrm{mg}$ decrease in the immediate pool size after Benemid administration. At least two explanations might be offered for this. One is that this "extra" uric acid is not derived exclusively from the immediate pool but from a more remote pool of uric acid. At present there does not seem 
to be any good way of testing this hypothesis. Another explanation is that with the renal tubules blocked, more exogenous uric acid finds its way into the urine. This hypothesis cannot be rigorously proved, but there are isolated facts that tend to support it. One is that subjects on Benemid continue to excrete "extra" uric acid as long as they continue taking the drug. Since the decrease in the serum uric acid concentration and the decrease in pool size occur within a day or two of the beginning of therapy, it would seem that the body would soon reach a state of minimum uric acid content and that thereafter "extra" uric acid would of necessity arise from external or dietary sources. Normally some uric acid does arise from dietary sources since it has been shown in this laboratory (3) that different dietaries, specifically high-fat as against high-protein diets, cause a significant difference in uric acid excretion.

The labeled uric acid that was injected into the non-gouty subject was not completely recovered as urinary uric acid. By day 162 no more $\mathrm{N}^{15}$ uric acid from the first injection was recoverable from the urine; hence it was assumed that all the injected labeled uric acid had already left the body pool. Since 28.5 per cent of the injected dose had

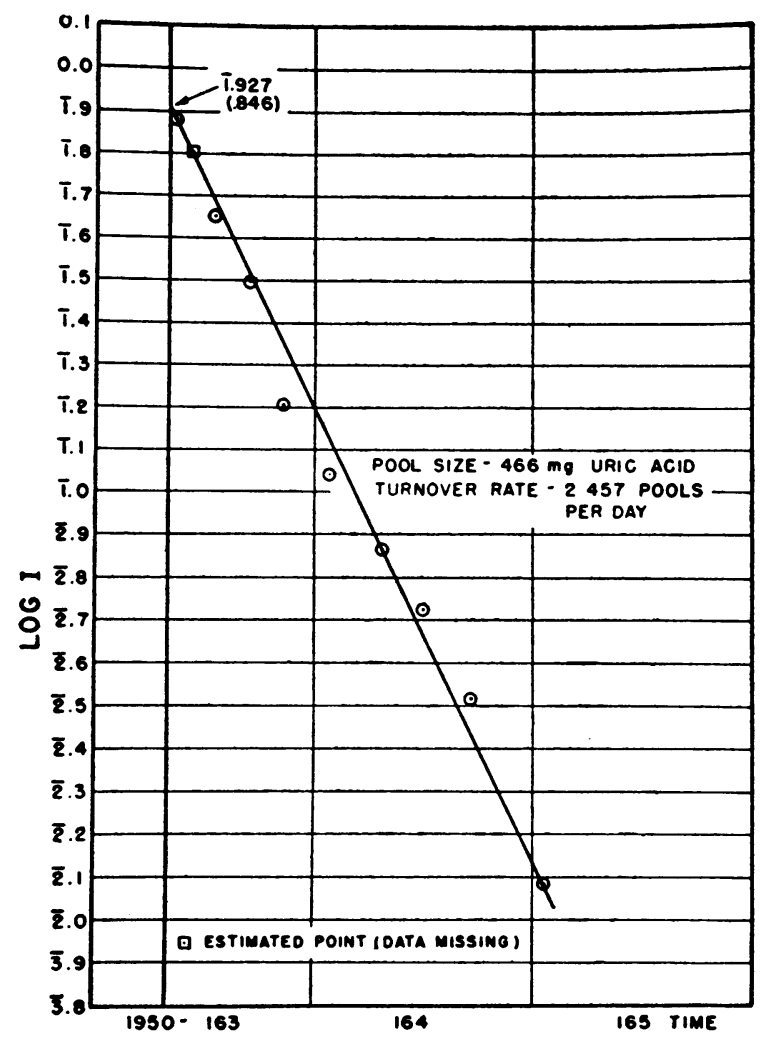

Fig. 2. Isotope Concentration of Urinary Uric Acid of Subject J. H. T. During Benemid Ingestion

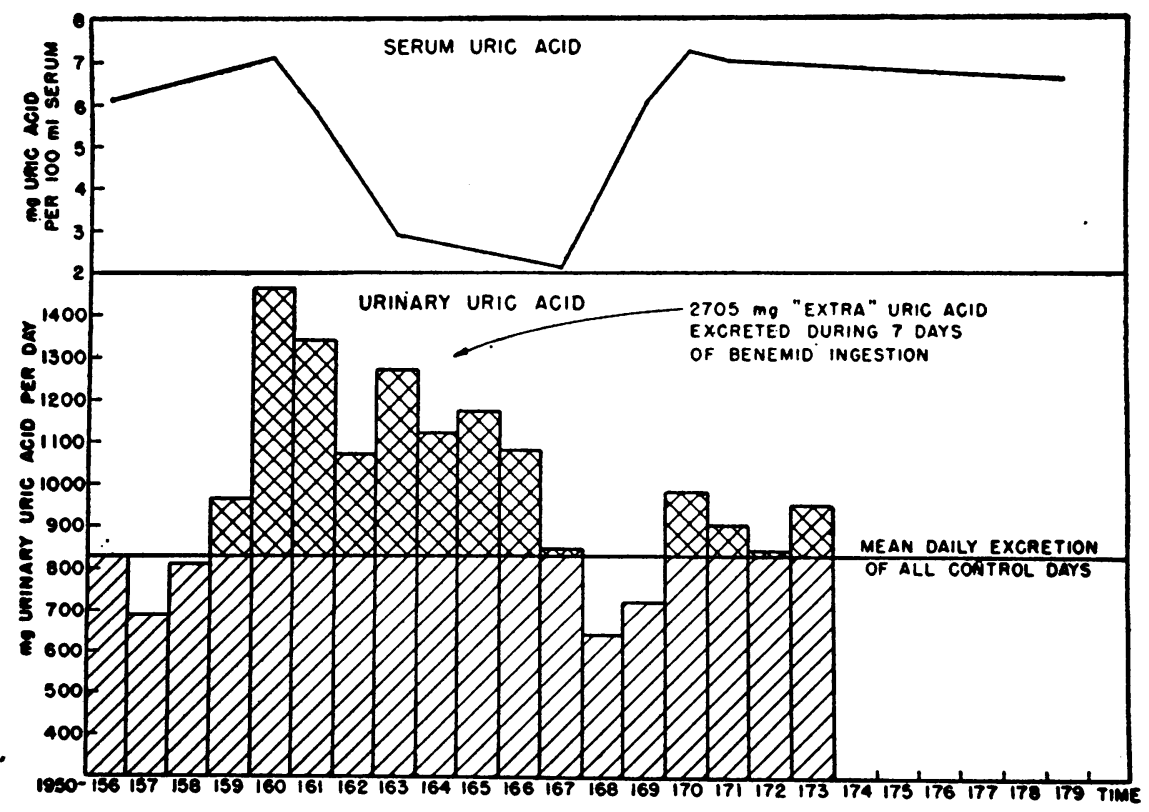

Fig. 3. Serum Uric Acid Concentration and Urinary Uric Acid Excretion of Subject J. H. T. Before, During, and After Benemid Ingestion 
not been accounted for as urinary uric acid, it was assumed that this amount had been disposed of by another route, probably catabolism since there has been scattered evidence for such a process $(4,5)$.

Before and during the second $\mathrm{N}^{15}$ uric acid injection period the subject had been taking Benemid. The injected uric acid was excreted quickly, and 89.6 per cent was recovered as urinary uric acid. This is consistent with the hypothesis that one or more alternate mechanisms is competing for the uric acid that would otherwise be excreted by the kidney. If the $\mathrm{N}^{15}$ concentration of the body uric acid pool is quickly reduced, the amount of the injected uric acid that can escape by the alternate routes will be reduced. Benemid does cause such a rapid decrease in the $\mathrm{N}^{15}$ uric acid concentration of the body pool, presumably by blocking resorption of urinary uric acid.

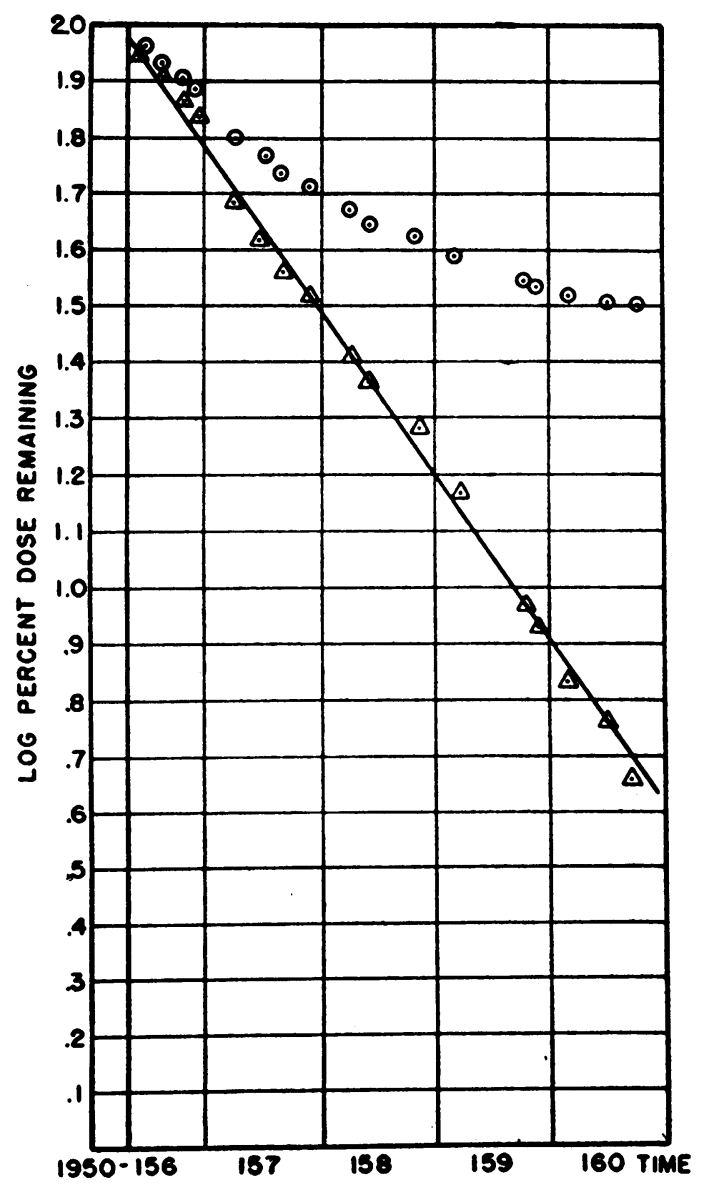

Fig. 4.

Subject J. H. T.-See text for explanation.

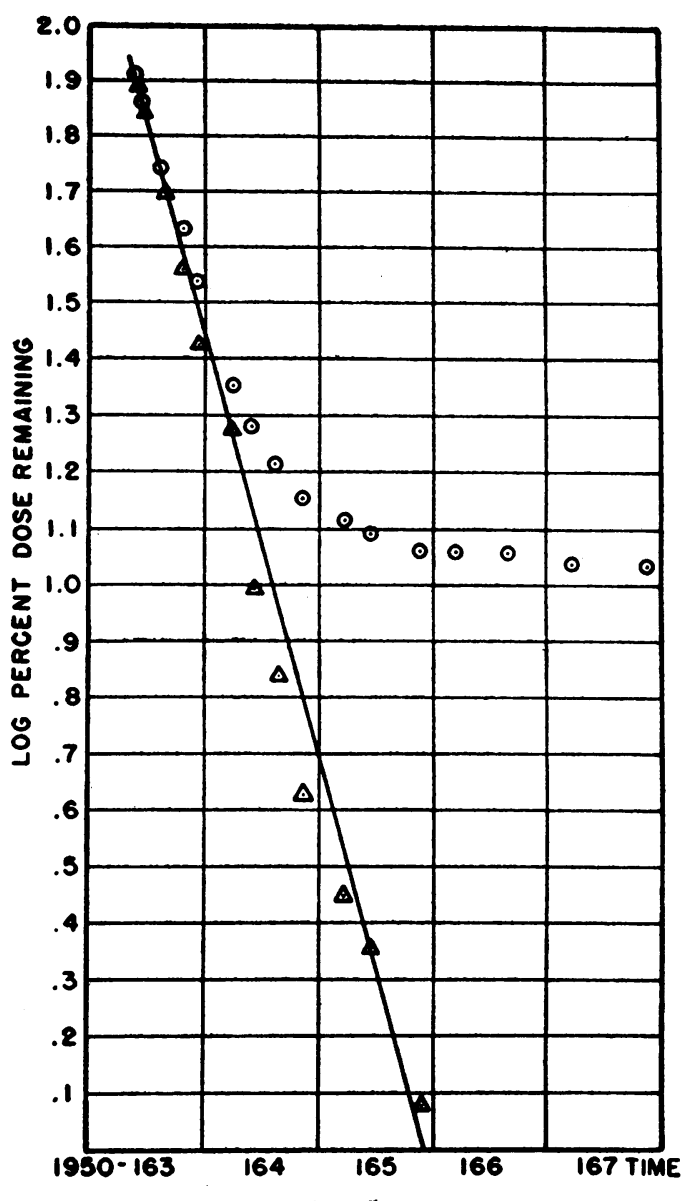

FIG. 5.

Subject J. H. T.-See text for explanation.

Further evidence for more than one pathway of uric acid disposal is presented in Figures 4 and 5. When the logarithm of the percentage of the dose apparently remaining in the body was plotted against time, the curve was not a straight line, neither during the control period (upper curve, Figure 4) nor during the Benemid period (upper curve, Figure 5). On the assumption that the amount of $\mathrm{N}^{15}$ uric acid remaining in the body pool should be calculated on the basis of only the uric acid excreted by the kidney, the data were recalculated. The amount of uric acid unaccounted for at the end of the study was subtracted from each datum and the logarithms of these values were again plotted in Figures 4 and 5 (lower curves). These new curves are straight lines. Thus the rate of urinary excretion of the labeled uric acid was directly related to the amount of the labeled uric acid actually left, not to the amount 
merely unaccounted for on the basis of urinary excretion.

The second subject of this study, W. S., although suffering from gouty arthritis, responded to Benemid therapy in much the same way as the first subject. Benemid ingestion was begun on day 181 and continued through day 186. As can be seen from Figure 6, the uric acid pool size in his control period was $2205 \mathrm{mg}$ and the turnover rate was .484 pools per day. After Benemid therapy (Figure 7) his pool size fell to $1622 \mathrm{mg}$ and the turnover rate rose to 1.006 pools per day. This paralleled a marked drop in serum uric acid concentration as shown in Figure 8. Yet the drop in the size of the immediate pool was only 573 mg while the "extra" uric acid excreted during the six days on Benemid was $4889 \mathrm{mg}$ as can be seen from the same figure. It is quite possible, especially in the gouty subject, that some of this "extra" uric acid is derived from a pool much larger than the immediate pool. There is also the suspicion that at least part of the "extra" uric acid is derived from dietary sources.

One striking difference between the behavior of the non-gouty and gouty subjects was the amount of the injected dose of isotopic uric acid recovered as urinary uric acid. In the gouty subject this was 98.9 per cent as can be seen from Table I. This

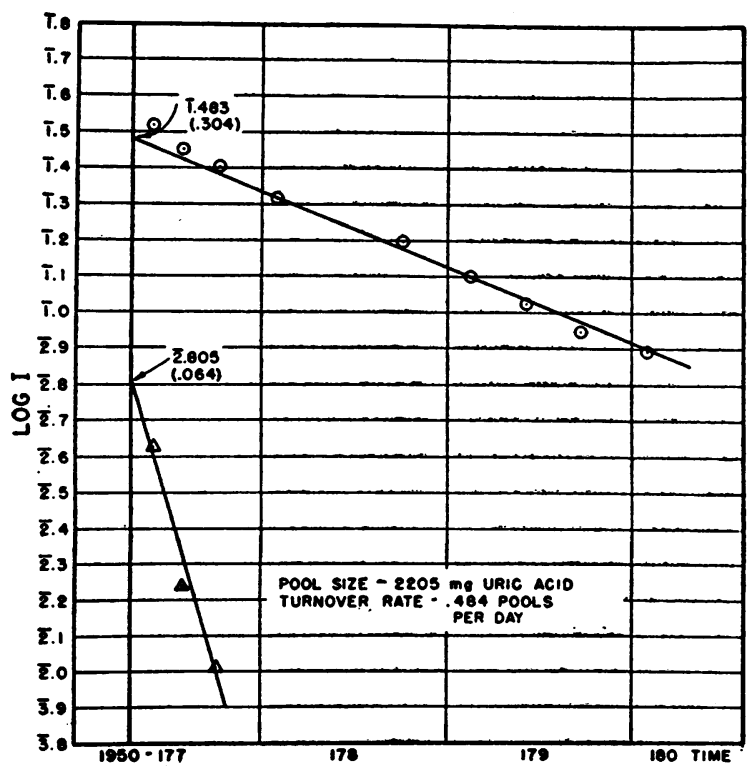

Fig. 6. Isotope Concentration of Urinary Uric Acid of Subject W. S. Before Benemid Ingestion

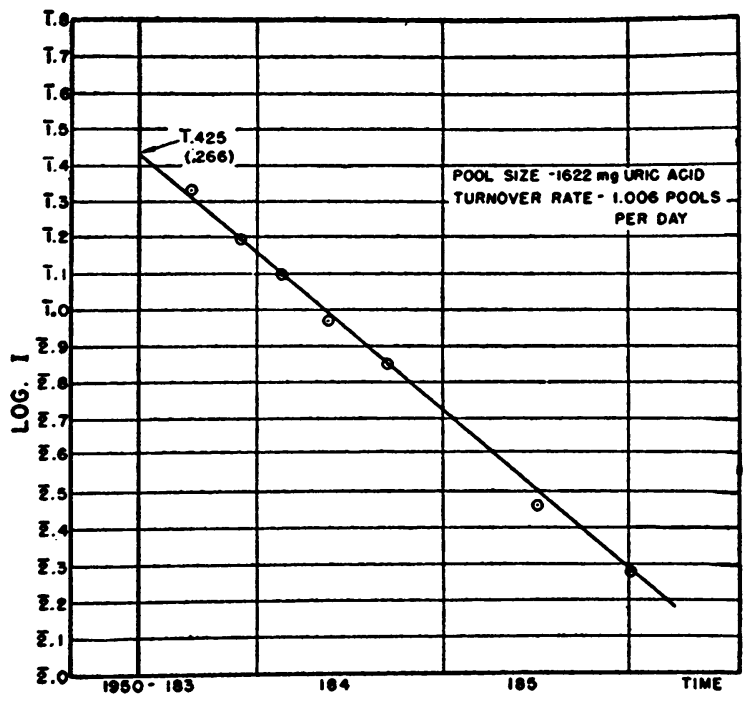

Fig. 7. Isotope Concentration of Urinary Uric AcId of Subject W. S. During Benemid Ingestion

subject was still excreting a small amount of labeled uric acid when the study was terminated, so that the ultimate recovery might have been even higher.

Since this subject apparently did not catabolize any uric acid, it became feasible to calculate his pool size at various times. In this study the ini-

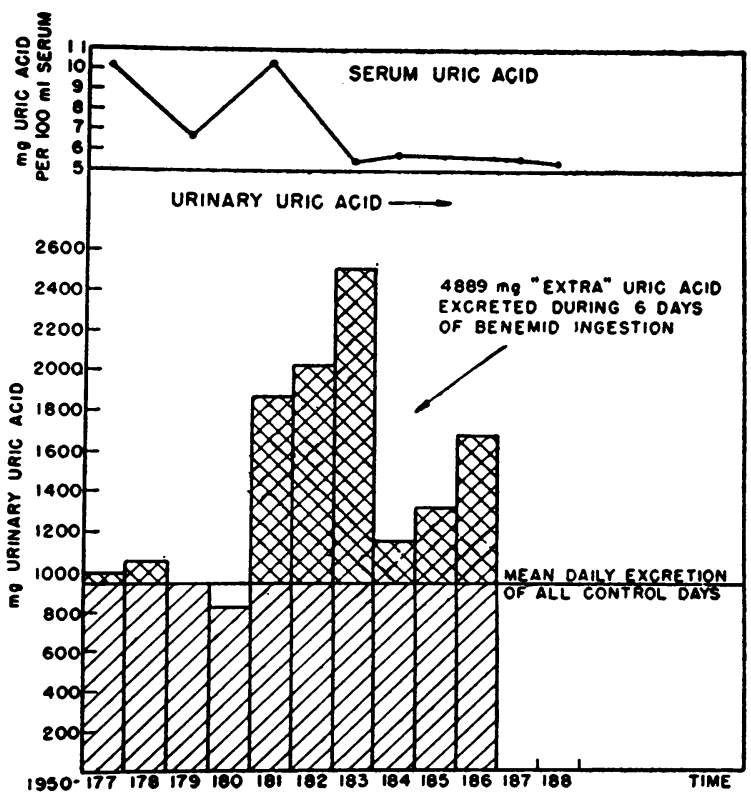

Fig. 8. Serum Uric Acid Concentration and UriNary Uric Acid Excretion of SubJect W. S. Before AND During Benemid Ingestion 
TABLE I

Uric acid pool size in gouty subject at successive intervals both before and during Benemid therapy

\begin{tabular}{|c|c|c|c|c|c|c|}
\hline Sample & $\begin{array}{l}\text { Time at } \\
\text { end of Sample }\end{array}$ & $\mathbf{M g} \times \mathbf{I}$ & \multicolumn{2}{|c|}{$\begin{array}{l}\text { Amount Left } \\
\mathrm{Mg} \times 1 \quad \%\end{array}$} & $\begin{array}{l}\text { Estimated I } \\
\text { at end of } \\
\text { Period }\end{array}$ & $\begin{array}{l}\text { Pool Size } \\
\text { (mg uric acid) }\end{array}$ \\
\hline $1 *$ & 177.312 & 0 & $831 * *$ & 100.0 & & \\
\hline $2-3$ & 528 & 71 & 760 & 91.5 & .299 & 2540 \\
\hline 4 & 649 & 74 & 686 & 82.6 & .274 & 2500 \\
\hline $5-6$ & 917 & 53 & 633 & 76.2 & .229 & 2760 \\
\hline $7-8$ & 178.271 & 55 & 578 & 69.5 & .191 & 3020 \\
\hline $9-10$ & 531 & 61 & 517 & 62.3 & .168 & 3080 \\
\hline $11-13$ & 179.003 & 73 & 444 & 53.4 & .133 & 3340 \\
\hline $14-15$ & 281 & 21 & 423 & 50.9 & .116 & 3640 \\
\hline $16-17$ & 545 & 28 & 395 & 47.5 & .103 & 3840 \\
\hline $18-20$ & 913 & 36 & 359 & 43.2 & .086 & 4180 \\
\hline $21-22$ & 180.267 & 21 & 338 & 40.7 & .072 & 4700 \\
\hline $23-26$ & 948 & 36 & 302 & 36.4 & & \\
\hline $27-28$ & 181.281 & 7 & 295 & 35.6 & & \\
\hline $29-35$ & 182.229 & 62 & .233 & 28.0 & & \\
\hline $36-43$ & 183.264 & 30 & 203 & 24.4 & & \\
\hline $44-45$ & 503 & 233 & $386 * * *$ & 31.0 & .236 & 1635 \\
\hline $46-47$ & 792 & 122 & 264 & 21.2 & .177 & 1490 \\
\hline $48-49$ & 184.024 & 68 & 196 & 15.7 & .140 & 1400 \\
\hline 50 & 243 & 45 & 151 & 12.1 & .112 & 1350 \\
\hline $51-52$ & 531 & 39 & 112 & 9.0 & .084 & 1330 \\
\hline $53-55$ & 875 & 41 & 71 & 5.7 & .059 & 1200 \\
\hline $56-57$ & 185.243 & 7 & 64 & 5.1 & .041 & 1560 \\
\hline $58-61$ & 778 & 21 & 43 & 3.5 & .024 & 1790 \\
\hline $62-64$ & 186.240 & is & 32 & 2.6 & .016 & 2000 \\
\hline $65-71$ & 187.240 & 18 & 14 & 1.1 & .008 & 1750 \\
\hline$S$ & collected imn & liately & ior to & tial in & tion of isotc & uric acid \\
\hline$*$ & injection: & $\mathrm{mg}_{\mathrm{g}} \times 1$ & 618 ato & $m$ perce & $t$ excess $=83$ & \\
\hline$* * *$ & injection: & $\begin{array}{l}m g \times 1 \\
+203\end{array}$ & $\begin{array}{l}618 \text { atc } \\
\text { emaini }\end{array}$ & $\begin{array}{l}\text { perce } \\
3 \text { from }\end{array}$ & $\begin{array}{l}\text { excess }=416 \\
\text { st injection) }\end{array}$ & 19 \\
\hline
\end{tabular}

tial dose minus the $\mathrm{N}^{15}$ uric acid excreted must equal the amount of $\mathrm{N}^{15}$ uric acid remaining in the body. These calculations were carried out in arbitrary units, since this simplified the calculations. The product of the number of milligrams of uric acid excreted during the first period and its isotope concentration in atoms per cent excess was subtracted from the product of the number of milligrams of uric acid in the injected dose and its isotope concentration. This new value, when divided by the estimated isotope concentration of the body pool at the end of this first period equalled the pool size in milligrams (neglecting the amount of uric acid contributed by the injected dose). The amount of isotopic uric acid excreted in the second period was similarly calculated and subtracted from the amount left at the end of the first period. This quantity divided by the estimated isotope concentration at the end of the second period gave the pool size at the end of the second period. As is apparent from Table $I$, the pool size increased constantly up to the point where it was no longer possible to make a satisfactory estimate of the isotope concentration of the body pool. When the pool size was plotted against time no asymptotic tendencies were evident so that an ultimate pool size could not be calculated. This may be due to the fact that in the body there are relatively large amounts of slowly equilibrating uric acid, perhaps in a solid phase.

In the Benemid period, the pool size started at a value closely approximating the pool size calculated by the graphical method and fluctuated down and up. This did not necessarily mean that there were not more than $1,600 \mathrm{mg}$ of uric acid in the whole body at this time. It may be that no appreciable amounts of labeled uric acid had an opportunity to equilibrate with the less accessible depots (tophi, bound phases) of body uric acid because of the rapidity with which the $\mathrm{N}^{15}$ uric acid was excreted by the blocked tubules. The tendency of the uric acid pool size to increase toward the end of this study was not immediately explainable, but the last two or three values should 
not be emphasized because the error of the isotope determination in this region is difficult to assess.

\section{SUMMARY}

One normal and one gouty subject have been injected intravenously with $\mathrm{N}^{15}$ labeled uric acid. The isotope concentration of the urinary uric acid was determined and the amount of urinary uric acid excreted was determined by the isotope dilution method. After a suitable control period each subject received oral daily doses of $2 \mathrm{gm}$ of Benemid (p-[di-n-propylsulfamyl]-benzoic acid), a potent uricosuric agent, and subsequently were reinjected with $\mathrm{N}^{15}$ labeled uric acid.

In the normal and the gouty subjects the amount of body uric acid that would immediately equilibrate with injected $\mathrm{N}^{15}$ uric acid decreased markedly after Benemid therapy, and the turnover rate of the body uric acid pool increased greatly.

The amount of "extra" uric acid that was excreted during the period of Benemid therapy was much greater than the amount that disappeared from the immediate pool. Some of this "extra" uric acid, especially in the gouty subject, might have come from a more remote depot of uric acid but other evidence suggests that the diet, under the conditions of this experiment, may have contributed to this "extra" uric acid.

In the control period of the non-gouty subject only 71.5 per cent of the injected $\mathrm{N}^{15}$ uric acid was recovered as urinary uric acid up to the time when its excretion ceased. During Benemid therapy the recovery of a second dose of $\mathrm{N}^{15}$ uric acid was 89.6 per cent. This was explained as being due to the more rapid excretion of the $\mathrm{N}^{15}$ uric acid in the second period, thus decreasing the time during which the labeled uric acid could be disposed of by a competing catabolic or other disposal route. The recovery of injected $\mathrm{N}^{15}$ uric acid in the total of both periods of the gouty subject was 98.9 per cent up to the time when excretion of the isotope had virtually ceased. This gouty subject ap- parently did not catabolize uric acid or dispose of it in any other way than by urinary excretion.

Since in the gouty subject all of the injected labeled uric acid was excreted as urinary uric acid, it was possible to calculate the uric acid pool size at succeeding time periods. The size of the pool in the control period of this subject rose from approximately $2,700 \mathrm{mg}$ at the time of injection of the labeled uric acid to $4,700 \mathrm{mg}$ within three days. It could not be reliably estimated thereafter. After Benemid therapy the pool size fluctuated around $1,600 \mathrm{mg}$.

Benemid was shown, both in the gouty and nongouty subject, to be a potent uricosuric agent, this action being associated with a reduction in the serum urate concentration and a reduction in the pool size of body uric acid.

\section{ACKNOWLEDGMENT}

The authors acknowledge with pleasure the assistance of Dorthea Tamborski who performed many of the determinations, and Frank Stein and Ernest Lehmann of the Physics Department of the University of Buffalo, who contributed to the operation of the mass spectrometer.

\section{REFERENCES}

1. Bishop, C., Garner, W., and Talbott, J. H., Pool size, turnover rate, and rapidity of equilibration of injected isotopic uric acid in normal and pathological subjects. J. Clin. Invest., 1951, 30, 879.

2. Folin, O., The preparation of sodium tungstate free from molybdate, together with a simplified process for the preparation of a correct uric acid reagent (and some comments). J. Biol. Chem., 1934, 106, 311.

3. Wilson, D., Bishop, C., and Talbott, J. H., A factorial experiment to test the effect of various types of diets on uric acid excretion in normal human subjects. To be published.

4. Folin, O., Berglund, H., and Derick, C., The uric acid problem. An experimental study on animals and man including gouty subjects. J. Biol. Chem., 1924, 60,361 .

5. Benedict, J. D., Forsham, P. H., and Stetten, D., Jr., The metabolism of uric acid in the normal and gouty human, studied with the aid of isotopic uric acid. J. Biol. Chem., 1949, 181, 183. 